\title{
Biosimilar Drugs for Inflammatory Bowel Disease: Is Similar Good Enough?
}

\author{
Mariangela Allocca $^{1} \cdot$ Gionata Fiorino $^{1} \cdot$ Silvio Danese $^{1}$
}

Published online: 3 August 2017

(C) Springer Science+Business Media, LLC 2017

Over 1 million subjects with immune-related diseases, including inflammatory bowel disease (IBD), have been treated with the therapeutic monoclonal antibody infliximab (IFX) since it was approved for clinical use. Soon after the expiration of the patent for originator IFX, the IFX biosimilar CT-P13 was approved for the same indications with the expectation of similar efficacy and safety, with significant cost savings.

The process of production of therapeutic monoclonal antibodies (biologics) is very complex. Since these molecules are produced in living organisms, they can vary according in terms of secondary, tertiary and quaternary structure and also have varying glycosylation patterns. Therefore, the production of a biosimilar needs to be carefully monitored in order to ensure that any differences in the characteristics of the molecule do not impact on the bioequivalence of the biosimilar in terms of immunogenicity and pharmacokinetics [1].

At the time of the approval of the IFX biosimilar CTP13 by the European Medicine Agency (EMA), there were no data on the use of this biosimilar in IBD. The only clinical studies were two randomized controlled trials in rheumatoid arthritis and ankylosing spondylitis subjects, with a follow-up period of 104 weeks, showing no differences in efficacy, safety and immunogenicity between the IFX biosimilar CT-P13 and the IFX originator, both in naïve and in patients previously treated with infliximab $[2,3]$. Due of the lack of data generated in IBD patients, the European Crohn's Colitis Organization (ECCO) had a

Silvio Danese

sdanese@ hotmail.com

1 Division of Gastroenterology, IBD Center, IRCCS Istituto Clinico Humanitas ICH, 20089 Rozzano, Milan, Italy cautious approach about the use of IFX biosimilar in IBD, especially with regard to switching from the originator to a biosimilar [4].

In this issue of Digestive Diseases and Sciences, Smits et al. reported the results of a prospective study of 83 IBD patients (57 Crohn's disease [CD], 24 ulcerative colitis [UC] and 2 IBD-unspecified [IBD-U] that switched from branded IFX (originator) to the IFX biosimilar CT-P13, after which they were followed for 1 year [5]. Both the disease activity, evaluated by Harvey-Bradshaw Index (HBI) for CD and Simple Clinical Colitis Activity Index (SCCAI) for UC and IBD-U, and the inflammatory biomarkers, including C-reactive protein (C-RP) and fecal calprotectin (FCP) did not change during the follow-up year. At week 52, the median IFX trough levels (TL) were not different from those at week 0. Seven patients developed antidrug antibodies (ADA), five at the baseline, and two during the follow-up period. Sixty-eight patients completed the 1-year of follow-up, 15 discontinued the IFX biosimilar CT-P13. In total, 11/83 patients (13\%) stopped the treatment, 5 for loss of response, and 6 for adverse events, namely two for skin rash and four for arthralgia. Despite some methodological limitations, such as the lack of a control group that continued receiving the IFX originator, and the heterogeneity of the study population due to the real-life study design, no significant differences from what would be expected from the originator were found. In particular, the rate of discontinuation of the IFX biosimilar for loss of response or occurrence of adverse events (13\%) is similar to those previously reported for IFX originator (10\%) [6]. Finally, regarding TL and ADA of 11 subjects who discontinued the IFX biosimilars, only 4 subjects developed ADA. In conclusion, this study helps confirm the bioequivalence of the IFX biosimilar CT-P13 in IBD patients treated with IFX originator. 
The increasing use of IFX biosimilars is expected to reduce costs from $€ 10$ to $€ 335$ million in the next 5 years [7]. Furthermore, other biosimilars will be launched in the market in the next years, such as biosimilars of adalimumab. Cost savings may change therapeutic strategies in immune-related diseases, including IBD. More patients may gain earlier access to more effective therapies and newer treatment regimens, such as individualized therapeutic schedules or combination therapy with two or more biologics with different mechanisms-of-action. Despite these clear advantages, real-life data on efficacy, safety, and immunogenicity of biosimilars are needed through long-term clinical studies, post-marketing vigilance, and disease-specific registries for monitoring adverse events and support the widespread use of biosimilars with significant cost savings.

\section{References}

1. Schiestl M, Stangler T, Torella C, Cepeljnik T, Toll H, Grau R. Acceptable changes in quality attributes of glycosylated biopharmaceuticals. Nat Biotechnol. 2011;29:310-312.
2. Yoo DH, Hrycaj P, Miranda P, et al. A randomised, double-blind, parallel-group study to demonstrate equivalence in efficacy and safety of CT-P13 compared with innovator infliximab when coadministered with methotrexate in patients with active rheumatoid arthritis: the PLANETRA study. Ann Rheum Dis. 2013;72:1613-1620.

3. Park W, Hrycaj P, Jeka S, et al. A randomised, double-blind, multicentre, parallel-group, prospective study comparing the pharmacokinetics, safety, and efficacy of CT-P13 and innovator infliximab in patients with ankylosing spondylitis: the PLANETAS study. Ann Rheum Dis. 2013;72:1605-1612.

4. Danese S, Gomollon F. ECCO position statement: the use of biosimilar medicines in the treatment of inflammatory bowel disease (IBD). J Crohns Colitis. 2013;7:586-589.

5. Smits LJT, Grelack A, Derikx LAAP, et al. Long-term clinical outcomes after switching from Remicade ${ }^{\circledR}$ to biosimilar CT-P13 in inflammatory bowel disease. Dig Dis Sci. (Epub ahead of print). doi:10.1007/s10620-017-4661-4.

6. Schnitzler F, Fidder H, Ferrante M, et al. Long-term outcome of treatment with infliximab in 614 patients with Crohn's disease: results from a single-centre cohort. Gut. 2009;58:492-500.

7. Fiorino G, Fazio M, Danese S. Biosimilar therapies in inflammatory bowel disease: should we care about patient profile? Expert Rev Clin Immunol. 2016;12:361-363. 